\title{
Breast reconstruction rate and profile in a Singapore patient population: a National University Hospital experience
}

\author{
Nadia $\underline{\operatorname{Sim}}^{1}$, MBBs, Sharon Soh${ }^{1}$, MBBS, Chuan Han $\underline{\text { Ang }}{ }^{2}$, MBBS, MRCs, Chor Hoong $\underline{H i n g}^{1,3}$, BN, AdvDip, \\ Han Jing Lee $\underline{\underline{H}}^{3,4}$, MBBS, MRCS, Vigneswaran Nallathamby ${ }^{3,4}$, MB BCh BAO LRCP/SI, MRCS, Yan Lin $\underline{\text { Yap }}^{4}$, MBBS, MRCS, \\ Wei Chen $\underline{\mathrm{Ong}}^{4}$, MBBS, MRCS, Thiam Chye $\underline{\mathrm{Lim}}^{4}$, MD, FRCS, Jane $\underline{\mathrm{Lim}}^{4}$, MBBS, FRCS
}

INTRODUCTION Breast reconstruction is an integral part of breast cancer management with the aim of restoring a breast to its natural form. There is increasing awareness among women that it is a safe procedure and its benefits extend beyond aesthetics. Our aim was to establish the rate of breast reconstruction and provide an overview of the patients who underwent breast reconstruction at National University Hospital (NUH), Singapore.

METHODS We evaluated factors that impact a patient's decision to proceed with breast reconstruction, such as ethnicity, age, time and type of implant. We retrospectively reviewed the medical records of women who had breast cancer and underwent breast surgery at NUH between 2001 and 2010.

RESULTS The breast reconstruction rate in this study was $24.3 \%$. There were 241 patients who underwent breast reconstruction surgeries (including delayed and immediate procedures) among 993 patients for whom mastectomies were done for breast cancer. Chinese patients were the largest ethnic group who underwent breast reconstruction after mastectomy $(74.3 \%)$. Within a single ethnic patient group, Malay women had the largest proportion of women undergoing breast reconstruction (60.0\%). The youngest woman in whom cancer was detected in our study was aged 20 years. Malay women showed the greatest preference for autologous tissue breast reconstruction (92.3\%). The median age at cancer diagnosis of our cohort was 46 years.

CONCLUSION We noted increases in the age of patients undergoing breast reconstruction and the proportion of breast reconstruction cases over the ten-year study period.

Keywords: breast, breast reconstruction, mammoplasty, reconstruction

\section{INTRODUCTION}

Breast cancer is the most common malignancy among women in Singapore (29\%) $)^{(1)}$ and worldwide (25\%). ${ }^{(2)}$ Breast reconstruction is now an integral part of breast cancer management. ${ }^{(3,4)}$ It aims to create a symmetrical, natural-looking breast mound. Since Vincent Czerny's first attempt at breast reconstruction in 1895, ${ }^{(5)}$ many techniques and methods have evolved to the modern-day standards of safety and aesthetics. Now, the complete treatment of breast cancer with reconstruction achieves two different goals of breast cancer management - oncologic management and aesthetic restoration. The increasing popularity of breast reconstruction can be attributed to factors such as improvements in surgical techniques, and changes in patient attitudes and preferences.

Studies have also shown that the decision to undergo breast reconstruction varies substantially by ethnicity, especially in the United States (US). ${ }^{(6-8)}$ Unlike many of its Southeast Asian counterparts, Singapore has a multiethnic population composed primarily of four ethnic groups: Chinese $(74.3 \%)$; Malay (13.4\%); Indian (9.1\%); and other ethnicities (3.2\%). ${ }^{(9)}$ In such an ethnically diverse population, the effect of various epidemiological factors has not been well studied. Currently, breast reconstruction data is lacking in the Southeast Asian region, including Singapore.

Our study aimed to establish the rate of breast reconstruction and provide an overview of those who underwent postmastectomy breast reconstruction at National University Hospital $(\mathrm{NUH})$, Singapore - a key institute that provides specialist services for breast reconstruction in Singapore.

\section{METHODS}

This retrospective study was carried out on all women who were diagnosed with breast cancer and underwent surgeries (mastectomy and subsequent breast reconstruction procedures) at NUH over a ten-year period (2001-2010). Patient information was collected from the hospital's main clinical information system (or the Computerised Patient Support System), scanned medical records and clinical files. The list of patients was generated from the NUH breast cancer registry database. Data of interest included the total number of reconstruction cases annually and patient sociodemographics, such as age and ethnicity (Chinese, Malay, Indian and other ethnicities). Data collection was done with approval from the National Healthcare Group Domain Specific Review Board. Computer software for statistical calculations,

${ }^{1}$ NUS Yong Loo Lin School of Medicine, National University of Singapore, ${ }^{2}$ Department of General Surgery, Singapore General Hospital, ${ }^{3}$ Department of Surgery, National University Health System, ${ }^{4}$ Division of Plastic, Reconstructive and Aesthetic Surgery, Department of Surgery, National University Health System, Singapore

Correspondence: Dr Jane Lim, Clinical Director and Senior Consultant, Division of Plastic, Reconstruction and Aesthetic Surgery, Department of Surgery, National University of Singapore, Level 8, NUHS Tower Block, 1E Kent Ridge Road, Singapore 119228. cfslimj@nus.edu.sg 
such as IBM SPSS Statistics version 22.0 (IBM Corp, Armonk, NY, USA), was used to generate the breast reconstruction rate.

\section{RESULTS}

Among the 1,686 surgeries performed, 993 were mastectomies and 693 were breast-conserving surgeries. Of the 993 mastectomies, there were 241 subsequent procedures for breast reconstruction (which included both immediate and delayed procedures). The overall breast reconstruction rate at NUH from 2001 to 2010 was $24.3 \%$ (241/993 patients). Table I shows the ethnic distribution of the women who underwent surgeries for breast cancer treatment and those who had subsequent breast reconstruction, as well as the age at cancer diagnosis of women who underwent breast reconstruction. The ethnic distributions of the women who underwent breast cancer surgery were similar to those who underwent subsequent breast reconstruction. Of those who underwent breast reconstruction, women of Chinese ethnicity constituted the largest ethnic group of patients $(74.3 \%)$.

Out of 241 breast reconstruction procedures, the majority ( $n=233,96.7 \%$ ) were immediate reconstructions, while the rest $(\mathrm{n}=8,3.3 \%$ ) were delayed reconstructions. For all eight patients who underwent delayed reconstruction, the procedures were performed within the ten-year time frame $(<1$ year, $\mathrm{n}=1$; 2 years, $\mathrm{n}=2 ; 3$ years, $\mathrm{n}=1 ; 4$ years, $\mathrm{n}=1 ; 5$ years, $\mathrm{n}=2$; 7 years, $n=1$ ). We noted a fivefold increase in the proportion of women who underwent breast reconstruction over the ten-year period, from $8.1 \%(10 / 123)$ in 2001 to $41.1 \%$ (44/107) in 2010 (Table II \& Fig. 1).

Within a single ethnic patient group, the largest proportion of women who underwent breast reconstruction after mastectomy were of Malay ethnicity $(60.0 \%)$, followed by women of Chinese (25.1\%), Indian (7.6\%) and other $(16.9 \%)$ ethnicities (Fig. 2). Similarly, within a single ethnic patient group, Malay women showed the greatest preference for the use of autologous tissue during breast reconstruction (92.3\%), while the corresponding proportions were $80.4 \%, 72.7 \%$ and $91.7 \%$ for women of Chinese, Indian and other ethnicities, respectively (Fig. 3).

The median age of all patients who underwent breast reconstruction was 46.0 years (Table I). The higher proportion of Malay women who underwent breast reconstruction within a single ethnic patient group was correspondingly associated with a significantly younger median age at cancer detection (40.0 years) among this ethnic group. In comparison, women of other ethnic groups had a higher median age at cancer diagnosis - Chinese (47.0 years), Indian (45.0 years) and other ethnicities (44.5 years) (Table I). Over the ten-year study period, we noted an increase in the age of women who underwent breast reconstruction. This was depicted by a relatively lower median age (40.5 years) in 2002 and a relatively higher median age (49.0 years) in 2009 (Table II).

Table I. Patients who underwent breast cancer surgery and reconstruction by ethnicity.

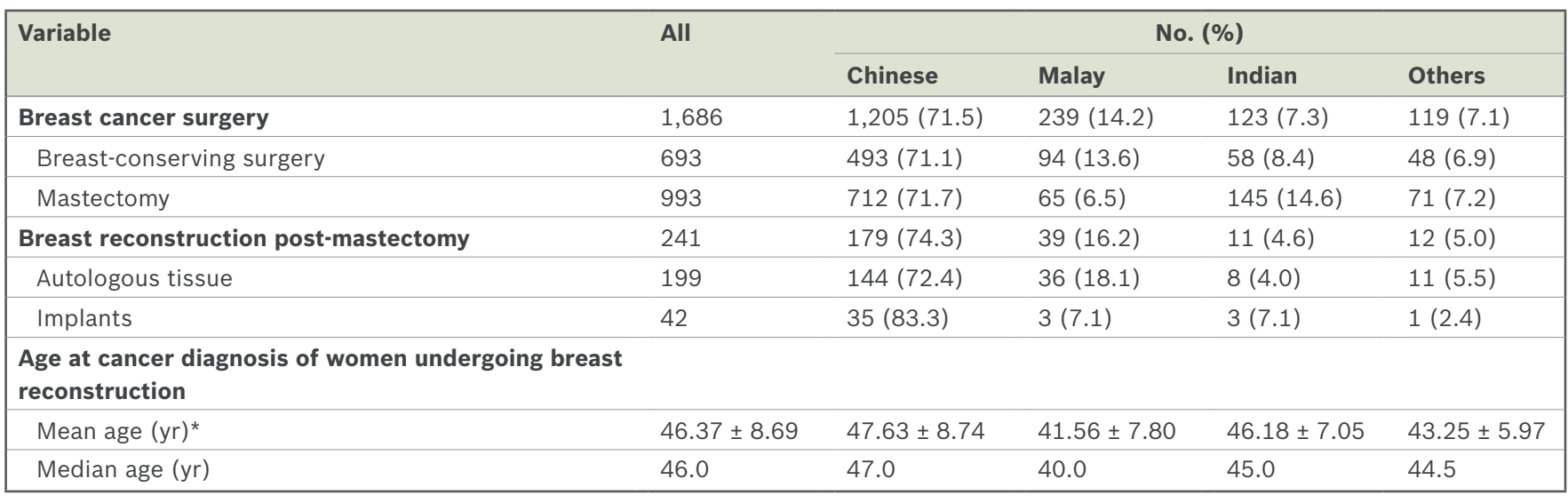

*Data presented as mean \pm standard deviation.

Table II. Patients who underwent breast cancer surgery and reconstruction by year.

\begin{tabular}{|c|c|c|c|c|c|c|c|c|c|c|}
\hline \multirow[t]{2}{*}{ Variable } & \multicolumn{10}{|c|}{ No. (\%) } \\
\hline & 2001 & 2002 & 2003 & 2004 & 2005 & 2006 & 2007 & 2008 & 2009 & 2010 \\
\hline Mastectomy $(n=993)$ & $123(12.4)$ & $125(12.6)$ & $104(10.5)$ & $91(9.2)$ & $67(6.7)$ & $79(8.0)$ & $91(9.2)$ & $86(8.7)$ & $120(12.1)$ & $107(10.8)$ \\
\hline $\begin{array}{l}\text { Subsequent breast } \\
\text { reconstruction } \\
(n=241)\end{array}$ & $10(8.1)$ & $20(16.0)$ & $13(12.5)$ & $13(14.3)$ & $14(20.9)$ & $22(27.8)$ & $28(30.8)$ & $34(39.5)$ & $43(35.8)$ & $44(41.1)$ \\
\hline $\begin{array}{l}\text { Median age of women } \\
\text { who underwent breast } \\
\text { reconstruction (yr) }\end{array}$ & 45.5 & 40.5 & 43.0 & 47.0 & 44.0 & 47.5 & 48.0 & 46.0 & 49.0 & 48.0 \\
\hline
\end{tabular}




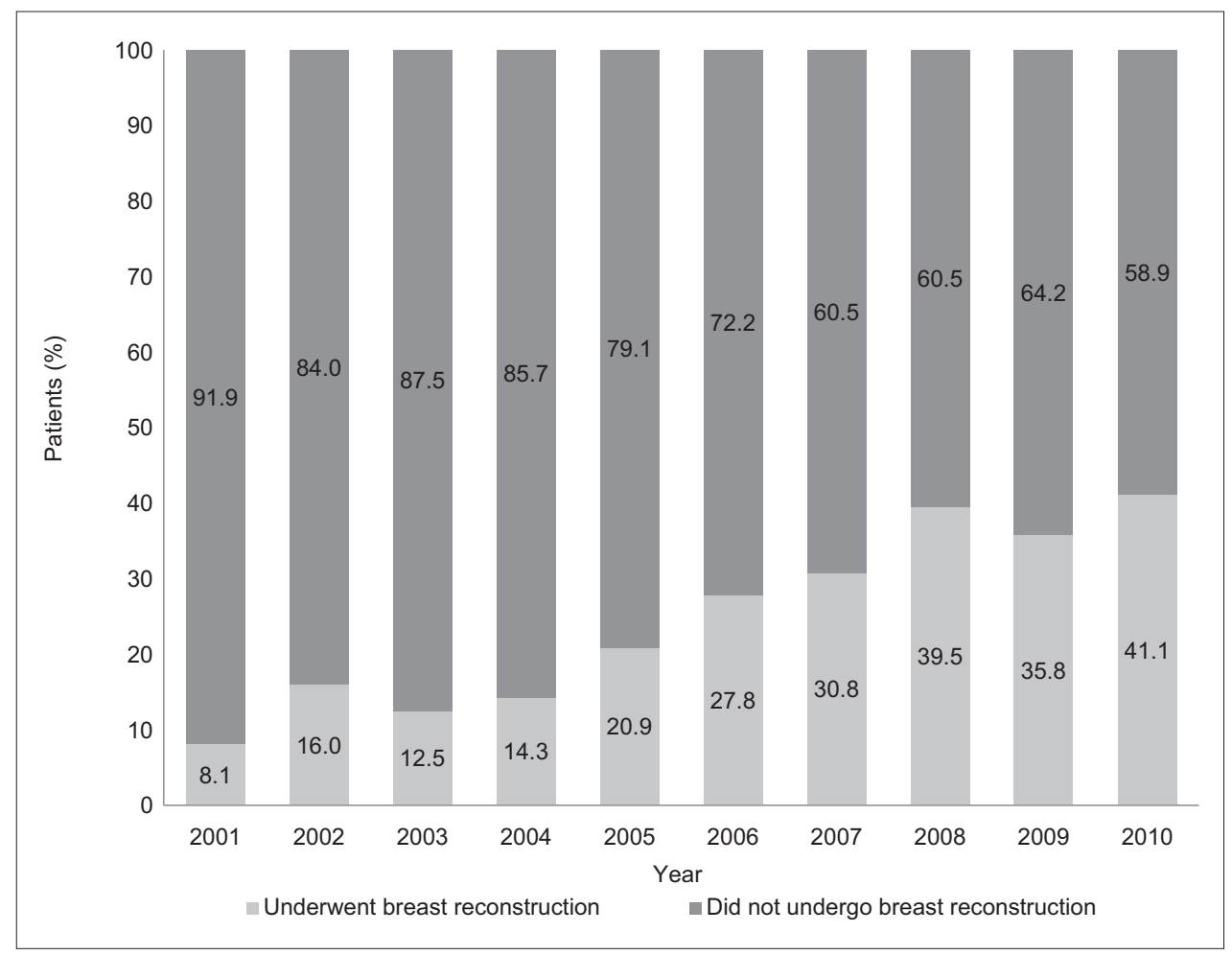

Fig. 1 Bar chart shows the proportion of women who underwent breast reconstruction after mastectomy by year $(n=241)$.

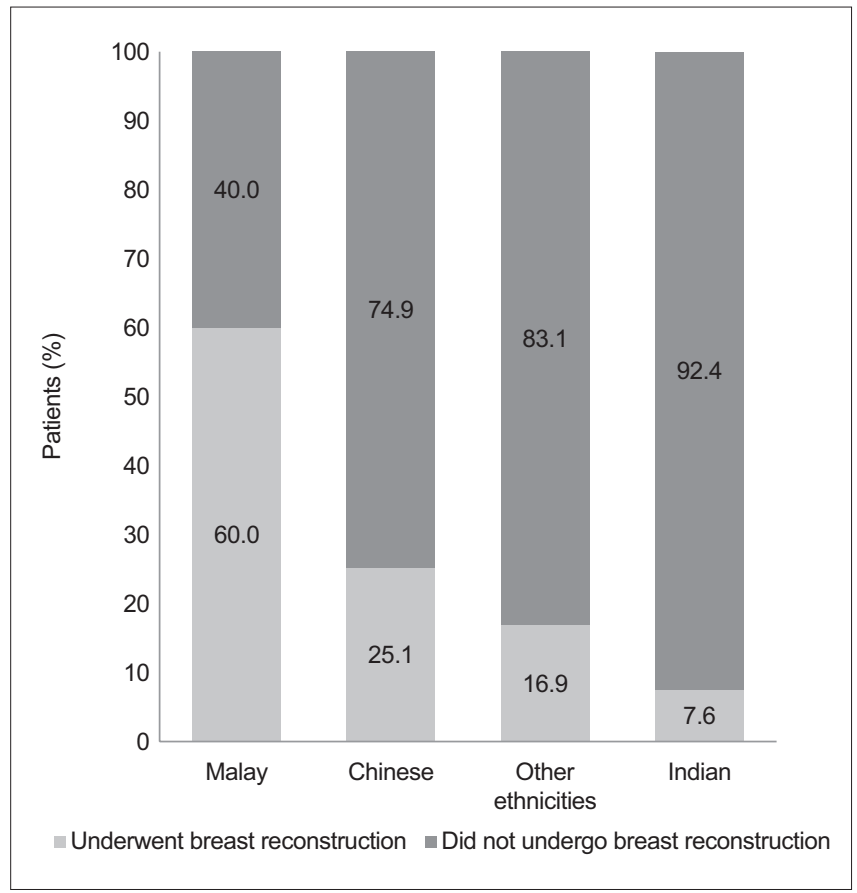

Fig. 2 Bar chart shows the proportion of women who underwent breast reconstruction after mastectomy by ethnic group $(n=241)$.

\section{DISCUSSION}

To our knowledge, this is one of the first studies to describe the rate of breast reconstruction at a large institution in Singapore. Our findings provide an overview of the profile of patients who underwent breast reconstruction surgery at NUH between 2001 and 2010.

The overall reconstruction rate at NUH was higher than those of other reports from Asia for a comparable time period. The

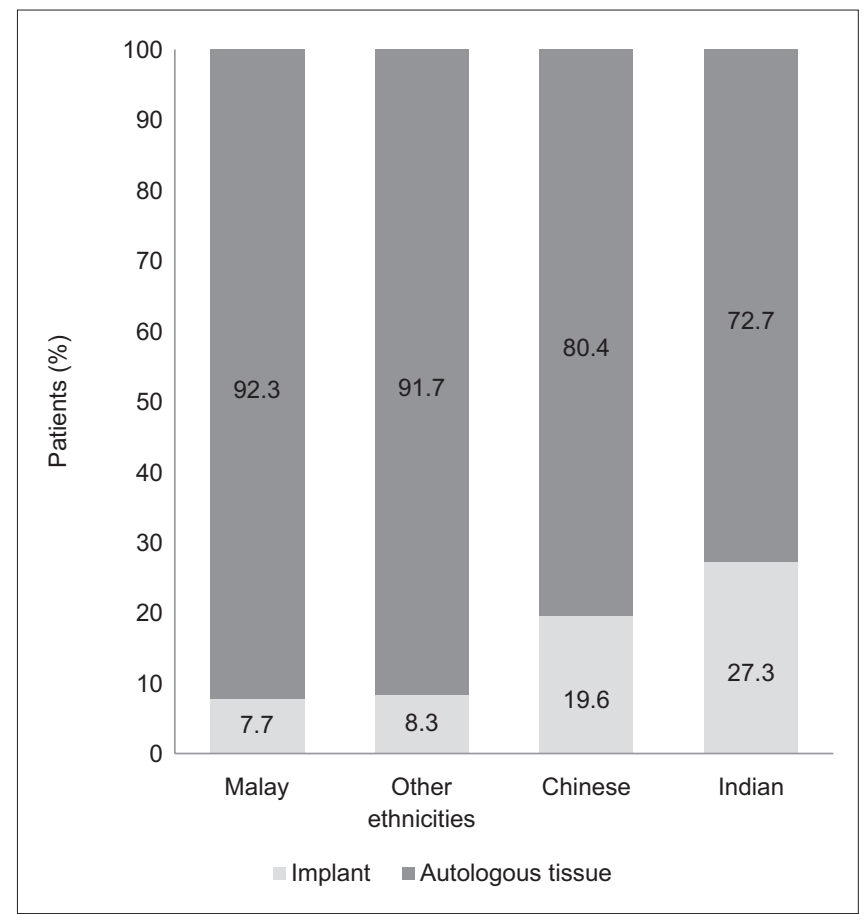

Fig. 3 Bar chart shows the proportion of women who opted for autologous tissue and implants for breast reconstruction by ethnic group $(n=241)$.

reconstruction rate at NUH was $24.3 \%$ in our study. The Korean Breast Cancer Society reported a $16.0 \%$ breast reconstruction rate in 2008 in Korea. ${ }^{(10)}$ Oda et al reported an immediate breast reconstruction rate of $11.2 \%$ in 2010 in over 1,300 hospitals in Japan. ${ }^{(11)}$ On the other hand, our breast reconstruction rate was comparable to those reported in Western populations. In the US, for instance, the breast reconstruction rate was $24.8 \%$ and $29.2 \%$ in 2003 and 2007, respectively. ${ }^{(12)}$ 
There was also a yearly increase in the percentage of breast reconstructions - a fivefold increase in the rate of breast reconstruction was seen, from $8.1 \%(n=10)$ in 2001 to $41.1 \%$ $(n=44)$ in 2010. The year 2010 saw the largest percentage of women undergoing breast reconstruction, which correlated with increased participation of women in the Singapore labour force, rising from $40.1 \%$ in 1999 to $43.8 \%$ in 2010. ${ }^{(13)}$ This finding may suggest that women were increasingly able to financially support themselves and their healthcare needs. This rising trend could also be attributed to increasing awareness of the benefits of breast reconstruction. Technological advancement has allowed women more convenient access to information on the Internet and from advocacy groups, such as the Singapore Breast Cancer Foundation. We opine that women in Singapore could have increasing levels of health literacy and comprehension of complex medical information, thus resulting in greater knowledge of the psychological, emotional and aesthetic benefits of breast reconstruction.

In Singapore, a multidisciplinary approach to the management of breast cancer is adopted to optimise surgical recovery and results, as seen in specialist centres such as NUH. Locally, breast surgeons work closely with plastic surgeons in co-managing patients with breast cancer. This is important, as it has been noted in local ${ }^{(14)}$ and US studies ${ }^{(15)}$ that variations in breast reconstruction rates may be linked to breast surgeons' attitudes and practice methods, as their perceptions of breast reconstruction could influence their decision to refer patients to plastic surgeons.

This close working relationship between breast surgeons and plastic surgeons in Singapore could explain the high breast reconstruction rate in our city-state, as compared to large countries, where the availability and distribution of medical resources are likely to vary geographically. Patients in Singapore have equal and convenient access to multidisciplinary healthcare, whereas patients in larger countries may be subject to differing regional policies or a lack of access to plastic surgery services in more rural areas. This has been reported in Japan, ${ }^{(11)}$ England $^{(16)}$ and Canada. ${ }^{(17)}$ A future survey of patients in Singapore would be helpful in delineating the relationship between surgeon referrals and ease of access to healthcare services and breast reconstruction.

Age has been widely reported as a predictor of breast reconstruction for women undergoing mastectomy for breast cancer. ${ }^{(18-20)}$ It is commonly thought that younger women tend to be more affected by the loss of their breasts and are thus more likely to receive reconstruction, ${ }^{(21)}$ whereas older women, particularly those of Asian descent, have "low demand for their body image". ${ }^{(22)}$ In our study cohort, the median age of breast reconstruction was 46 years, with the majority aged more than 46 years. This trend parallels breast cancer demographics locally and regionally, where a majority of patients with breast cancer are aged 45 years and above. ${ }^{(1,23)}$ Our findings may dispel the popular belief that younger women prefer reconstruction whereas older women do not.

Lipa et al showed encouraging data with regard to autologous reconstruction in older patients aged over 65 years. ${ }^{(24)}$ Veronesi et al found acceptable rates of complications, even with longer operating times, prolonged anaesthesia and greater fluid shifts. ${ }^{(25)}$ No significant difference was noted by Ludolph et al for minor or major complications in the postoperative period. ${ }^{(26)}$ Similar data was reported by Veronesi et al for implant-based reconstruction in older patients. ${ }^{(25)}$

It is possible that at a later stage of life, fewer women would consider breast reconstruction important. With a complex operative procedure for autologous reconstruction, possible comorbidities and prolonged recovery periods in older patients, there are also concerns that the risks might outweigh the benefits of reconstruction for older patients. However, our data showed an increase in the median age of reconstruction over the tenyear study period from 45.5 years in 2001 to 48.0 years in 2010, with the greatest age at 49.0 years in 2009. Does this suggest that increasing age is becoming less significant in the decision against breast reconstruction for both the patient and surgeon? A deeper and more extensive survey of our patient population might be necessary for a more comprehensive understanding of such associations.

Ethnically, we found that the largest proportion of patients who underwent reconstruction was Chinese $(74.3 \%)$; this was followed by Malay patients $(16.2 \%)$, those of other ethnicities (5.0\%) and Indian patients (4.6\%). This difference in reconstruction rate among women of different ethnicities is likely related to the disease pattern and ethnic composition of breast cancer in the local population - Chinese (80.8\%), Malay (11.3\%), Indian (5.9\%) and other ethnicities (2.0\%). ${ }^{(1)}$

In the present study, the uptake of reconstruction among Indian women was $7.6 \%$, which was the lowest among all the ethnic groups. We postulate that information on breast reconstruction may not be widely disseminated among Indian women.

Within a single ethnic patient group, the largest proportion of patients who underwent reconstruction was seen among Malays $(60.0 \%)$. This could be explained by the earlier diagnosis of breast cancer among Malay women, at the median age of 40 years, which was the lowest among all the ethnic groups. According to local cancer demographics, the median age at diagnosis for Malays is 46 years, which is also the lowest among the various ethnic groups, with Chinese patients at 51 years and Indian patients at 53 years. ${ }^{(1)}$ Regional studies have also found that Malay women tend to be diagnosed with breast cancer at a younger age with larger tumours and later stages than Chinese and Indian women. ${ }^{(27)}$ The development of breast cancer is multifactorial. Genetic and environmental factors may play a role in predisposing Malay women to developing breast cancer at a younger age. Further epidemiological and biochemical studies may be relevant in elucidating this predisposition.

Similar to a study by Simpson et al on the incidence of breast cancer among Filipino women in Canada, ${ }^{(28)}$ our finding may have a profound effect on future screening mammography guidelines and the ideal age to start breast cancer screening for women of Malay descent. According to current clinical practice guidelines in Singapore, normal-risk women are encouraged to attend breast cancer screening once in every two years. ${ }^{(29)}$ 
Malay patients $(92.3 \%)$ made up the greatest proportion of women who underwent autologous breast reconstruction in our study, with the remaining three out of 39 Malay patients $(7.7 \%)$ undergoing implant reconstruction. Within the other ethnic groups, $72.7 \%$ of Indian patients, $80.4 \%$ of Chinese patients and $91.7 \%$ of patients of other ethnicities underwent autologous breast reconstruction. The much higher proportion of Malay patients who underwent autologous reconstruction as opposed to implant reconstruction suggests the impact of culture and religion on medical perspectives, as women of different ethnic backgrounds tend to have different sets of cultural beliefs and expectations. Our results may suggest complex dynamics between cultural/religious preferences and a patient's decision to undergo reconstruction and the type of material used for reconstruction.

Our study had several limitations. First, only patients who underwent both breast resection and reconstruction surgeries at NUH were included in the study. Future analysis could integrate study populations from other surgical institutions in Singapore to obtain a more representative finding of the local population. Also, due to the retrospective nature of the study, we were unable to establish causality. Instead, we drew conclusions based on associations between the known factors and breast reconstruction in our cohort.

In conclusion, this present study provided an overview of the profile of patients who underwent breast reconstruction at a tertiary institution in Singapore. The information garnered has provided us with a better understanding of the factors that may be associated with a patient's decision to undergo breast reconstruction. This study also provides a platform for future epidemiological research to establish factors that are associated with post-mastectomy breast reconstruction.

\section{ACKNOWLEDGEMENT}

This research was done with approval from the National Healthcare Group Domain Specific Review Board (NUH/2010-0007).

\section{REFERENCES}

1. Singapore Cancer Registry Annual Registry Report Trends in Cancer Incidence in Singapore [online]. Singapore: National Registry of Diseases Office; 2015. Available at: https://www.nrdo.gov.sg/docs/librariesprovider3/defaultdocument-library/cancer-trends-report-2009-2013.pdf?sfvrsn=0. Accessed July 31, 2015.

2. Ferlay J, Soerjomataram I, Dikshit R, et al. Cancer incidence and mortality worldwide: sources, methods and major patterns in GLOBOCAN 2012. Int J Cancer 2015; 136:E359-86.

3. Platt J, Baxter N, Zhong T. Breast reconstruction after mastectomy for breast cancer. CMAJ 2011; 183:2109-16.

4. Rozen WM, Ashton MW, Taylor GI. Defining the role for autologous breast reconstruction after mastectomy: social and oncologic implications. Clin Breast Cancer 2008; 8:134-42.

5. Uroskie TW, Colen LB. History of breast reconstruction. Semin Plast Surg 2004; 18:65-9.
6. Alderman AK, Wei Y, Birkmeyer JD. Use of breast reconstruction after mastectomy following the Women's Health and Cancer Rights Act. JAMA 2006; 295:387-8.

7. Alderman AK, McMahon L Jr, Wilkins EG. The national utilization of immediate and early delayed breast reconstruction and the effect of sociodemographic factors. Plast Reconstr Surg 2003; 111:695-703.

8. Alderman AK, Hawley ST, Janz NK, et al. Racial and ethnic disparities in the use of postmastectomy breast reconstruction: results from a population-based study. J Clin Oncol 2009; 27:5325-30.

9. Department of Statistics, Singapore. Population Trends 2016 [online]. Available at: http://www.singstat.gov.sg/docs/default-source/default-documentlibrary/publications/publications_and_papers/population_and_population_ structure/population2016.pdf. Accessed May 3, 2018.

10. Ko SS; Korean Breast Cancer Society. Chronological changing patterns of clinical characteristics of Korean breast cancer patients during 10 years (1996-2006) using nationwide breast cancer registration on-line program: biannual update. J Surg Oncol 2008; 98:318-23.

11. Oda A, Kuwabara H, Fushimi K. Disparities associated with breast reconstruction in Japan. Plast Reconstr Surg 2013; 132:1392-9.

12. Kruper L, Holt $A, X u X X$, et al. Disparities in reconstruction rates after mastectomy: Patterns of care and factors associated with the use of breast reconstruction in Southern California. Ann Surg Oncol 2011; 18:2158-65.

13. Ministry of Manpower, Singapore. Singapore Workforce 2010. Available at: http://www.mom.gov.sg/newsroom/press-releases/2010/singaporeworkforce-2010. Accessed August 15, 2015.

14. Lim J, Low SC, Hoe M. Breast reconstruction after mastectomy: a survey of general surgeons in Singapore. ANZ J Surg 2001; 71:207-11.

15. Hawley ST, Hofer TP, Janz NK, et al. Correlates of between-surgeon variation in breast cancer treatments. Med Care 2006; 44:609-16

16. Jeevan R, Cromwell DA, Browne JP, et al. Regional variation in the use of immediate breast reconstruction after mastectomy for breast cancer in England. Eur J Surg Oncol 2010; 36:750-5.

17. Baxter N, Goel V, Semple JL. Utilization and regional variation of breast reconstruction in Canada. Plast Reconstr Surg 2005; 115:338-9.

18. Hall SE, Holman CD. Inequalities in breast cancer reconstructive surgery according to social and locational status in Western Australia. Eur J Surg Oncol 2003; 29:519-25

19. Hvilsom GB, Hölmich LR, Frederiksen K, et al. Socioeconomic position and breast reconstruction in Danish women. Acta Oncol 2011; 50:265-73.

20. Yu KD, Di GH, Wu J, et al. Development and trends of surgical modalities for breast cancer in China: a review of 16-year data. Ann Surg Oncol 2007; 14:2502-9.

21. Charavel M, Brémond A, Courtial I. Psychosocial profile of women seeking breast reconstruction. Eur J Obstet Gynecol Reprod Biol 1997; 74:31-5.

22. Jia-jian C, Nai-si H, Jing-yan X, et al. Current status of breast reconstruction in Southern China: A 15 year, single institution experience of 20,551 breast cancer patients. Medicine (Baltimore) 2015; 94:e1399.

23. Green M, Raina V. Epidemiology, screening and diagnosis of breast cancer in the Asia-Pacific region: current perspectives and important considerations. Asia Pacific J Clin Oncol 2008; 4 Suppl 3:S5-13.

24. Lipa JE, Youssef AA, Kuerer HM, Robb GL, Chang DW. Breast reconstruction in older women: advantages of autogenous tissue. Plast Reconstr Surg 2003; 111:1110-21.

25. Veronesi $\mathrm{P}$, Ballardini B, De Lorenzi $\mathrm{F}$, et al. Immediate breast reconstruction after mastectomy. Breast 2011; Suppl 3:S104-107.

26. Ludolph I, Horch RE, Harlander M, et al. Is there a rationale for autologous breast reconstruction in older patients? A retrospective single center analysis of quality of life, complications and comorbidities after DIEP or ms-TRAM flap using the BREAST-Q. Breast J 2015; 21:588-95.

27. Bhoo-Pathy $\mathrm{N}$, Hartman $\mathrm{M}$, Yip $\mathrm{CH}$, et al. Ethnic differences in survival after breast cancer in South East Asia. PLoS One 2012; 7:e30995.

28. Simpson JS, Briggs K, George R. Breast cancer amongst Filipino migrants: a review of the literature and ten-year institutional analysis. J Immigr Minor Health 2015; 17:729-36.

29. Ministry of Health, Singapore. Cancer Screening. $\mathrm{MOH}$ Clinical Practice Guidelines 1/2010. Available at: https://www.moh.gov.sg/content/dam/moh_ web/HPP/Doctors/cpg_medical/current/2010/cpg_Cancer\%20Screening\%20 Booklet\%20FINAL\%20v6.pdf. Accessed April 18, 2018. 